Document downloaded from:

http://hdl.handle.net/10251/159611

This paper must be cited as:

Moreno Cardenas, A.; Nakamura Pinto, MK.; Pietrosemoli, E.; Zennaro, M.; Rainone, M.; Manzoni, P. (2020). A Low-Cost and Low-Power Messaging System Based on the LoRa Wireless Technology. Mobile Networks and Applications (Online). 25(3):961-968. https://doi.org/10.1007/s11036-019-01235-5

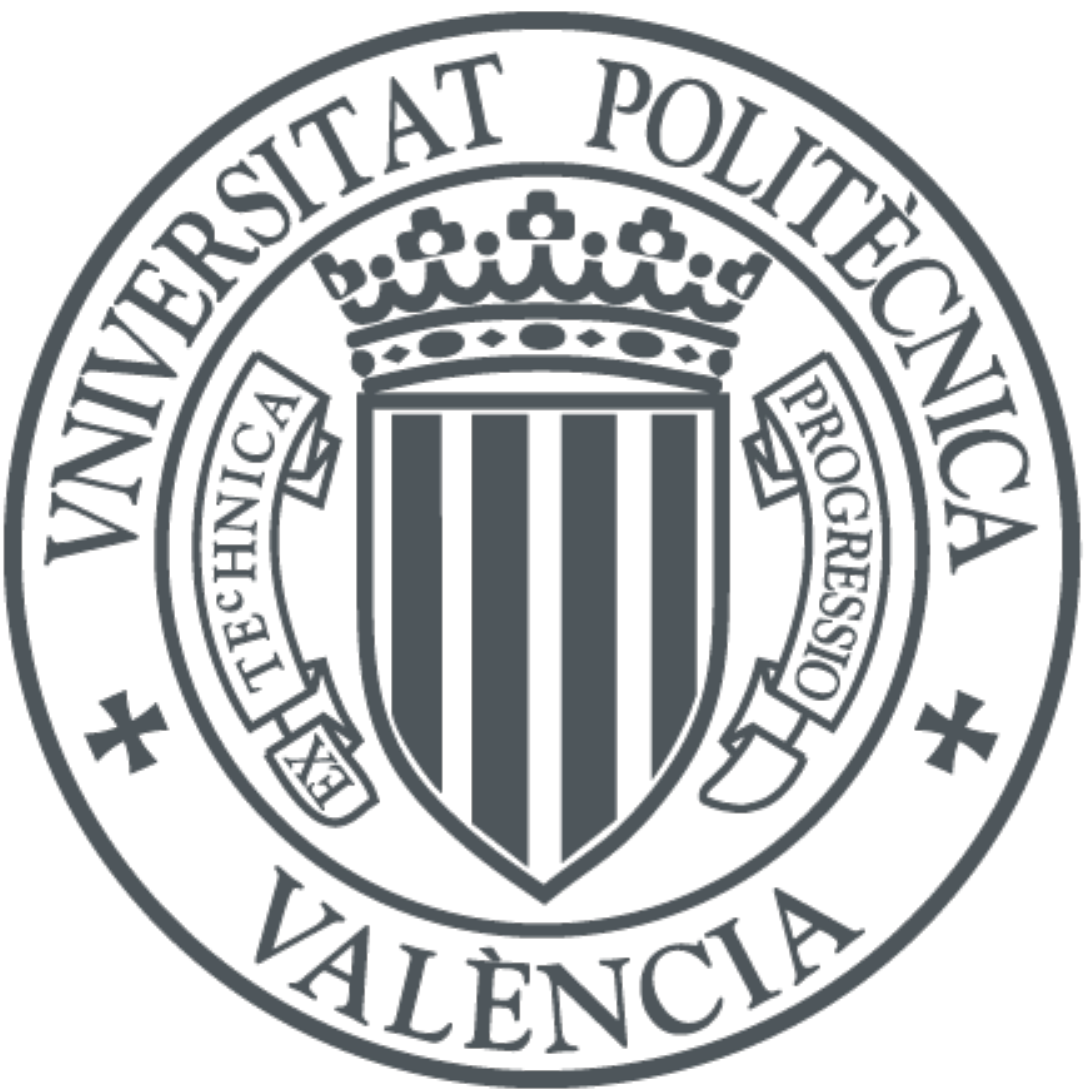

The final publication is available at

https://doi.org/10.1007/s11036-019-01235-5

Copyright Springer-Verlag

Additional Information 


\title{
A low-cost and low-power messaging system based on the LoRa wireless technology
}

\author{
Angelica Moreno Cardenas · Miguel \\ Kiyoshy Nakamura Pinto • Ermanno \\ Pietrosemoli • Marco Zennaro • Marco \\ Rainone • Pietro Manzoni
}

Received: date / Accepted: date

\begin{abstract}
In this paper we describe a low-cost and low-power consumption messaging system based on LoRa technology. More that one billion people worldwide cannot access even the most basic connectivity services. For them even simple messaging services would be of great help, for example to farmers wishing to know the price of goods they want to sell or buy before deciding whether a possibly long, expensive and exhausting trip is undertaken.

LoRa networks allow for very long wireless links that can connect villages and towns. This system falls in the category of community networks, where users build their own network where no commercial infrastructure is available. In addition to the simple messaging application, LoRa can be used to distribute sensor information to communities or to provide disaster alerts or meteorological data.
\end{abstract}

Keywords LoRa · Messaging · ICT4D · Community Networks

\section{Introduction}

A service like SMS has been the killer application for mobile services all over the world since it offered a flexible communication channel between individuals and among members of a community. The possibility to communicate between villages and between villages and main cities is a highly demanded service either for purely personal use or for commercial purposes in rural areas in developing countries, too.

For example, Martinez et al, offer in [6] an analysis of the communication needs in rural primary health care in developing countries. Although many interesting

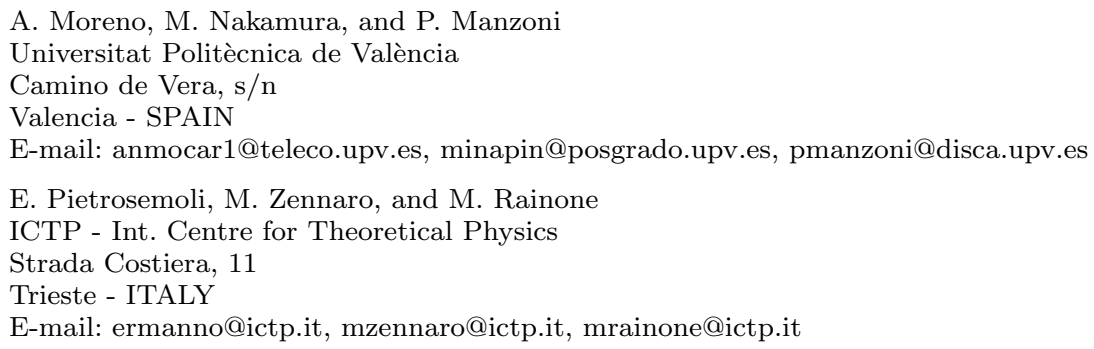


applications of telemedicine can be implemented with broadband solutions, one very simple application originally implemented over $\mathrm{HF}$ voice only radio communication proved quite successful: scheduling patient doctor's appointments. It was found that patients in isolated areas had to spend significant time and resources to reach the nearest hospital, and often they could not be treated immediately but given an appointment at a time that implied a second trip from home. A simple messaging application like the one we are proposing could serve to arrange for a specific date in which the patient is guaranteed treatment, thus saving time and resources.

The lack of coverage in rural areas is the consequence of a basic economic challenge: deploying infrastructure there can be twice as expensive, while revenue can be as much as ten times lower [8]. In this work we define the architecture for a messaging system that combines very cheap and flexible devices and the LoRa technology to establish links that can span wide areas with an easy to use interface. The unlicensed $868 \mathrm{MHz}$ or 433 bands in Europe and Africa as well as the $900 \mathrm{MHz}$ in America can be exploited for this purpose. By leveraging the LoRa modulation [9] transmission speed can traded for range. This possibility offers a versatile method for lowering the throughput in order to allow the data transfer to occur in very low $\mathrm{S} / \mathrm{N}$ instances, even with received signal power as low as 1 percent of the noise power.

In areas not covered by cellular service providers, very abundant in developing countries a few kilometres from towns and main roads, LoRa can provide a very low cost, community operated communication system that addresses many basic needs encompassing a variety of use cases. In mountainous regions one can leverage the terrain topography to accomplish line of sight transmissions at very long distances by fitting the nodes with external high gain antennas. A $316 \mathrm{~km}$ transmission using low-power nodes and small antennas is reported in [5] as an example of what can be achieved.

In this work we focus on a basic application of the LoRa system which is a SMSlike, one-to-one messaging. The low cost of LoRa devices, the use of unlicensed bands, and the the wide availability of WiFi-enabled devices (smart phones, tablets and PCs) that provide the platform for user interface, constitute the ingredients for installing a community network, built and maintained by the direct beneficiaries, after they are properly trained. Moreover, we integrated in our proposal a gateway device that, when Internet access is available, can forward messages to Telegram ${ }^{1}$ users.

Although this is very useful when there are no other systems in place, more applications can be developed using the same solution. For example, a Bulletin Board System (BBS) like functionality can be provided to inhabitants of isolated areas. It can be used to offer both weather forecasting as well as local data gathering if sensors are in place, health-care information, market prices of crops, and so on. In this case the communication is one-to-many allowing everyone to read the messages.

Finally, our proposed solution can also be leveraged to support ordinary LoRa applications, like environmental sensors, weather forecasting and so on. If these sensors are equipped with LoRa transceivers, they can be located beyond the reach of cellular towers and send data to the LoRa hub which can forward them to the

\footnotetext{
1 https://telegram.org/
} 
interested parties. In particular, for disaster mitigation text only communications using the LoRa device can be very valuable when the cellular service is disrupted and also to extend connectivity beyond the range of cellular towers.

The paper is organised as follows. Section 2 presents some of the most relevant solutions available in the literature. Section 3 presents the architecture of the proposed solution. Section 4 shows the results of the performance evaluation of a prototype of our proposal. Finally, Section 5 analyses the sustainability of the solution, and Section 6 presents the conclusions.

\section{Related Work}

Cognitive radio [10] is a growing technology that promises to overcome one of the most challenging problems of modern wireless communications, namely the scarcity of spectrum. Access to the radio spectrum is currently largely based on a fixed allocation, that is, different frequency bands are assigned to different services. With the vast increase in wireless applications and services in many countries, most of the available spectrum has been allocated. On the other hand, careful studies reveal that most of the assigned spectrum experiences low utilisation. Cognitive radio allows wireless networks to work without the need for dedicated spectrum. This possibility, in the context of rural communications, means that cognitive radio networks can be deployed at lower costs. The most notable example of a rural cognitive radio system is the IEEE 802.22 standard. Such technology offers two main advantages, that is, a dedicated spectrum is not required, and coverage is large. These two advantages help service providers address the cost-demand mismatch. The 802.22 technology is designed to provide an average coverage of 33 $\mathrm{km}$.

DakNet [1] is one of the first messaging system aimed at providing services beyond the coverage of GSM networks. This proposal leverages the use of a cyber cafe or a post office combining the existing transportation and communications infrastructure with a physical mean of transportation with wireless data transfer to extend the Internet connectivity. DakNet employs a delay tolerant infrastructure to offer applications like voice mail, e-mail and electronic bulletin board system (BBS) at much lower cost than their real-time counterparts. Short point to point links are created between kiosks and a portable device on board of a public transportation vehicle (for instance a bus) serving the area of interest. The bus contains a simple WiFi transceiver connected to a server and when in range of one of the outlying information kiosks it synchronises data for later processing. The end user access the updated information delivered to the kiosk by means of WiFi with their own phone or tablet.

A similar example was deployed in the rural area of South Africa [3]. This solution offers a multimedia content distribution system through Delay Tolerant Networks. The main use given is for Mobile movie entertainment, possibly combined with educational content for health care. The DTNs devices are based on infostations, which can be installed in taxis or mobile stations. Micro-entrepreneurs are also using infostations that allow them to access multimedia content to reproduce in their communities through a device known as cinema-in-a-backpack. Infostations communicate only with their direct neighbours through local radio communication such as wireless local area networks (WLAN), they act as peers collaborating 
to transmit the content. The content is downloaded first from a server to a fixed infostation, then the data is sent using an epidemic forwarding approach through the DTN network when the infostations are in the radio range. The system relies also on the taxis that are traveling, distributing content to micro-entrepreneurs with mobile infostations and fixed infostations at other taxi stations.

Bytewalla [7] allows the use of Android phones for the physical transport of data between network nodes in areas where no other links are available, or where existing links should be avoided for security reasons. In some areas, it is profitable, at least in the short-term perspective, to organize such physical data transport instead of implementing a physical network infrastructure, such as fiber optic cables or wireless broadband links. Mobile phones are by far the most commonly available mobile device, even in developing regions. The architecture of the Bytewalla network consists of two networks that can interoperate from two separate remote locations. The implementation of a storage and forwarding messaging application and a Sentinel surveillance health care application (SSA) running on Bytewalla represent some usage scenarios.

More recently, GreenLinks [2], was proposed as a platform providing mobile services, that are reliable and resilient to intermittency, supporting applications in rural contexts in extreme operating environments with little power and no cellular coverage. The basic element of the GreenLinks network is a Virtual Cell Node $(\mathrm{VCN})$ which is a programmable and open cellular base station. It allow the easy deployment of a base stations using low power software that works effectively in an IP Backplane. Each VCN requires access to a backhaul link and operates in a licensed spectrum band. GreenLinks allows a distributed VCN collection to interoperate as a single cellular network that can offer reliable, available, and resilient cellular services.

The examples above are just a few representing the growing area of ICT for development. This area aims at providing sustainable solution for ubiquitous connectivity . A fundamental reference document was recently produced by members of the IRTF group "Global Access to the Internet for All Research Group (GAIA)" [4] . The activities of GAIA are mainly oriented to create increased visibility and interest among the wider community on the challenges and opportunities to enable global Internet access, in terms of technology as well as in the social and economic drivers for its adoption.

We followed the experience of previous works and used the guidelines of the GAIA members to define an architecture for a messaging system that combines very cheap and flexible devices and the LoRa technology to establish links that can span wide areas with an easy to use interface. Moreover we integrated a gateway device that, when a link to Internet is available, can forward messages to Telegram users.

\section{The proposed architecture}

Figure 1 presents the overall architecture proposed . At the core there are dedicated devices, called hubs, that create the connectivity spot inside an area. The hubs must have both a WiFi (IEEE 802.1b/g/) and a LoRa transceiver.

The hubs work as standard WiFi access point to provide connectivity to close by devices. The interface with the messaging application is a web based page (see 


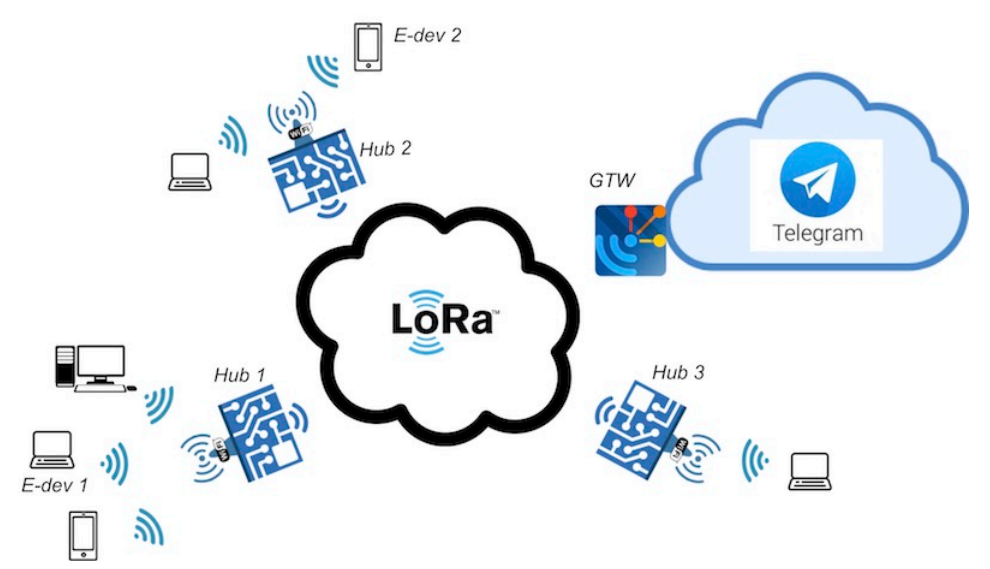

Fig. 1: Overall structure of the messaging platform.

Figure 2(a)). The user can decide whether to send a text a message to either a specific destination or to all reachable users, or to check for incoming messages stored in the hub. The hubs uses POST commands from the connected devices to either send a message or return previously received and locally stored ones.

Every user need to "register" before interchanging any message. Registration is required to allow the system to localise end-point which could have moved to a different location meanwhile. When a user sends a message, the local hub "learns" that that user is connected through it and creates an entry in a table. The first step is to discover where the destination user is located. To this end the hub sends a broadcast message using the physical layer LoRa protocol to all the surrounding devices. It then gets a response from the device which has that end user as a registered one.

Using a reliable protocol unicast messages are transferred and stored in the destination hub. Once the user to whom the message is addressed checks for available message he or she will receive the one stored in the local hub. The unicast protocol is based on a classical stop-and-wait ARQ approach with a dynamic and adaptive value for the retransmission delay. The protocol ensures that information is not lost due to dropped packets and that packets are received in the correct order.

The packet structure used by this protocol is very simple with two bits for control and a 32 bytes field to store the name to be searched. A broadcast user was included for messages that are to be delivered to all the registered users. The packet structure is shown in Figure 3.

We must point out that the maximum application payload depends on the selected data rate. For example, assuming the European $863-870 \mathrm{MHz}$ band, in worst propagation conditions, one should assume the lowest data rate, provided by SF12, where the node cannot send more than about 51 bytes per packet; with SF7, the payload might be 222 bytes. These values consider LoRaWAN protocol which adds at least 13 bytes to the application payload. The maximum packet size used was set according to the spreading factor used, i.e. 25 bytes for SF12 and 200 bytes for SF7, with a fixed 24 bytes header. 


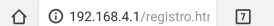

Kiyo

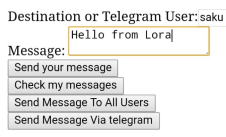

Back to home

(a) The Web based interface.

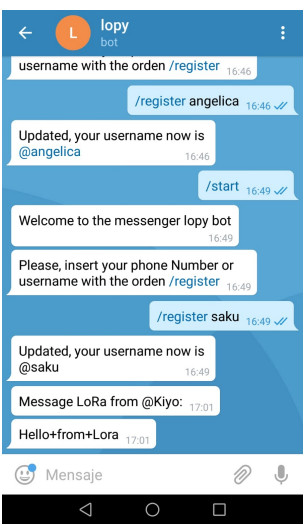

(b) The Telegram Bot.

Fig. 2: Screen capture of the web based interface (left) and of an interaction through Telegram (right)

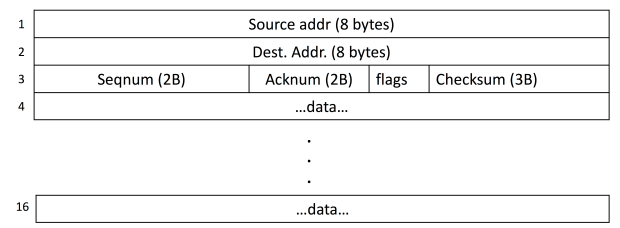

Fig. 3: Structure of the packet used by the stop-and-wait ARQ.

To better integrate our architecture with standard Internet application we designed a gateway hub to link it with Telegram ${ }^{2}$, a widely used messaging application (see Fig. 2(b)). We selected Telegram since if offers so called Bots, that is third-party applications that run inside Telegram. Users can interact with bots by sending them messages, commands and inline requests.

The gateway receives via LoRa messages directed to a Telegram user, registered through the Bot, and forwards that to the user's phone via the Internet link. The gateway of our prototype is based on a Raspberry Pi board provided with a Lora adaptor and a stable connection to the Internet. The processes required by the Bot are running on the Raspberry Pi.

For the creation of the Bot we have made use of the tool called BotFather, a Telegram complement that allows generating Bots by answering a set of simple questions and that provides a unique token that identifies the Bot.

The interaction mode between the Gateway and the LoPy device is exactly identical to the one described in the previous paragraph, that is, first an exchange of messages is carried out to confirm whether the user (in this case the Telegram user) is connected, then the message is sent. Since the device used for the prototype (a Raspberry $\mathrm{Pi}$ ) offered more possibilities, we made use of additional software

\footnotetext{
2 https://telegram.org/
} 
tools such as the inclusion of a database in mysql to record the user names along with the Id Telegram of each device to allow more efficient queries.

Finally, to ease management, additional commands were added to the bot to allow a better interaction, these are:

- /start: starts the interaction with the Bot;

- /register: to allow the registration or update of user names or phone numbers;

- /help: provides help on the available commands and their use.

\section{Experimental results}

In our experiments we used devices called LoPy ${ }^{3}$, a MicroPython enabled microcontroller, based on the latest Espressif ESP32 chipset. It has a dual processor and three transceivers (LoRa, WiFi, Bluetooth) and two antenna connectors: one for the $868 \mathrm{MHz}$ band used by LoRA and another for the $2.4 \mathrm{GHz}$ band used by WiFi and Bluetooth. This allows fitting the antenna best suited for the application. For instance, high gain directional antennas can be used to connect a rural village to a city that could be at a very long distance, using very small power by leveraging the spread spectrum features of LoRa modulation. The network processor handles the WiFi connectivity and the IP stack while the main processor is entirely free to run the user application. It has a 512KB RAM memory and allows for an external flash of 4MB. It has hardware floating point acceleration and can be programmed in Python multi-threading. A prototype of the complete hub, made of a LoPy node with an omnidirectional antenna, an external battery and a $5 \mathrm{~W}$ solar panel, is shown in Figure 4(a).

In the solution we propose our Gateway will be a Raspberry Pi with a LoRa module and TL-WN722N antenna. The Raspberry Pi will be responsible for receiving the messages via LoRa and sending them via by the Bot Telegram to registered users. The integration of these devices gives our system a greater scope, extending the coverage to all the internet users of Telegram. The prototype used is pictured in Figure 4(b).

Figure 5(a) shows the ordered set of outcomes of the user discovery process. The median value for the whole process is $2.077 \mathrm{msec}$. Once the user location is know, this process is not repeated.

We measured the performance of the system using a metric called "successful transfer time (STT)". It measures the transfer time of a message from the point of view of the sender. It is computed from the moment at which the first fragment of the message is sent, to the moment when the last ACK of the last fragment of the message is received.

Figure 5(b) shows the behaviour of the STT when varying the distance between two nodes. The median values are shown. Plots are obtained using a spreading factor (SF) of 7 and message size of 1,256 , and 512 bytes. As can be seen, there is an almost constant behaviour in the results although clearly the STT grows as the message size grows. It seems that the system is quite stable to the increase in distance and very few retransmissions were necessary during the experiment. Overall we had a retransmission rate of $9 \%$ but we must point out that the distribution of retransmissions was practically independent from the distance. The

\footnotetext{
3 https://pycom.io/
} 


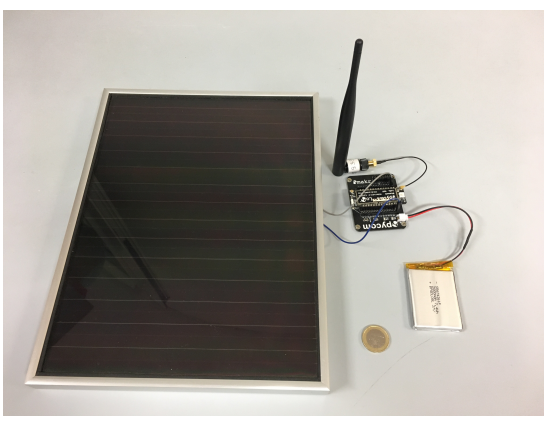

(a) Complete system.

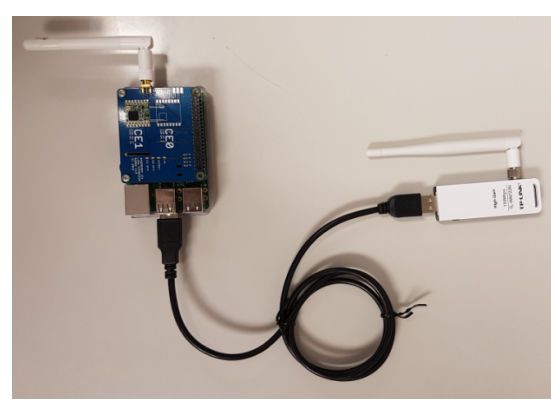

(b) A gateway.

Fig. 4: Prototypes of the system. On the left a complete system; notice the use of a solar panel to charge the battery. On the right a prototype used as a gateway. It is based on a RaspberryPi, a TP-Link antenna and a LoRa module

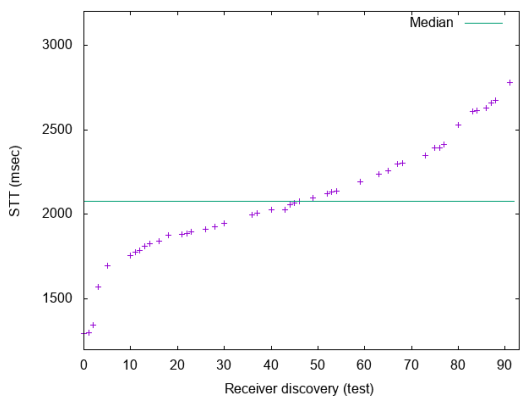

(a) user discovery process

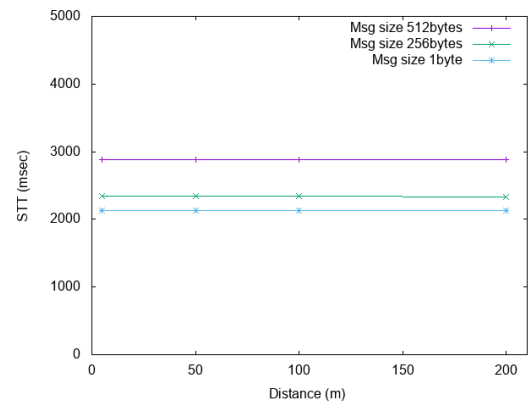

(b) STT versus distance between two nodes.

Fig. 5: On the left the user discovery process; ordered set of outcomes ("tests"). On the right the behaviour of the STT when varying the distance between two nodes (median values)

absolute values of the STT highlight that the set-up time required by the two devices to sync the transmitter has the highest overhead, followed by the actual data sending.

Figure 6(a) presents the scenario where we checked the behaviour of the system with greater distances than the experiments described above. The link was realised by connecting a device on the rooftop of one of the buildings of the ICTP research center in Trieste and the port area of Trieste. There is a clear line-of-sight over the $6.9 \mathrm{~km}$ path as shown in Figure 6(b).

The performance of the system was stable and almost identical to the case at shorter distances. Namely, the average value for sending a unicast message was $2173 \mathrm{~ms}$, which dropped to $1210 \mathrm{~ms}$ for broadcast messages. Retransmissions were rare events in the experiments but, when present, they clearly impacted on the average SST. SSTs of up to 10 seconds were measured. We think that these results do not affect the usability of the systems since we consider the waiting time more than reasonable. No effect on the delivery of the message was detected. 


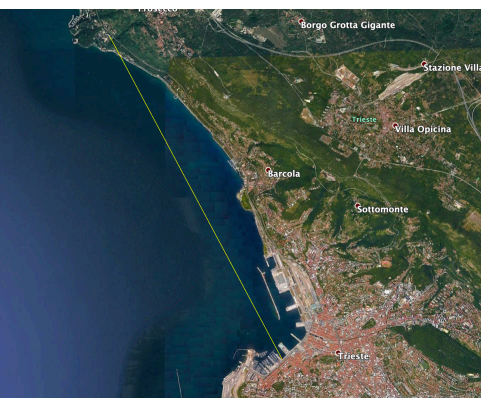

(a) Map view of the link trajectory.(Image obtained using Google Earth, (C)2019 DigitalGlobe)

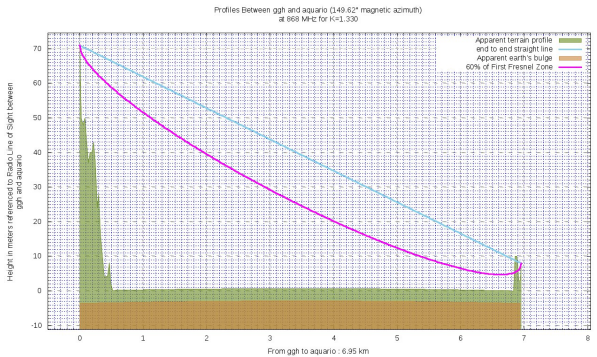

(b) Terrain profile between the two locations.

Fig. 6: Link between a device on the rooftop of one of the buildings of the ICTP research center in Trieste and the port area of Trieste. Figure (b) shows the profile of the $6.95 \mathrm{~km}$ link.

Figure 7(a) allows to better view the evolution of the STT as a function of the message size. As we can see, the STT clearly grows as the message size increases, while it is evident that the impact of distance is negligible.

Finally, Figure 7(b) shows the behaviour of the STT when varying the frequency of the generated messages, more exactly, when the number of generated simultaneous messages increases. Plots were obtained using a spreading factor of 7 and message size of 256 bytes. As can be seen the STT grows linearly with the number of simultaneous messages reaching the extreme case when 14 simultaneous users were trying to send a message; a maximum delay of 80 seconds was observed in a few cases. The median value for the 14 simultaneous users was $35.892 \mathrm{~s}$. With more than 14 simultaneous users the system showed a very unreliable behaviour and we considered it as basically unusable.

\section{Sustainability of the solution}

In order for any networking solution to be sustainable, several aspects have to be taken into account, namely: power consumption, cost, and regulation compliance.

We measured the power consumption of the proposed solution in two conditions: WiFi-only communication and WiFi+LoRa communication. In the first case (while compiling the message on the web interface) we measured $0.476 \mathrm{~W}$, in the second case (when sending a message via LoRa) we measured $0.587 \mathrm{~W}$, an increase of $23 \%$ in power consumption. With a maximum power consumption of $0.587 \mathrm{~W}$, the device can easily be powered via renewable sources such as solar. A $3.7 \mathrm{~V}$, $2000 \mathrm{mAh}$ battery can power the hub for 17 hours and a $5 \mathrm{~W}$ peak solar panel is enough to replenish the battery in most climates.

Our proposed solution requires a limited initial investment which can be shared among community members and does not imply any recurring costs. This is the well known model used in community networks, where wireless routers are installed in a community, which might not necessarily connected to the Internet. The proposed network architecture is based on LoRa devices which cost about 30 


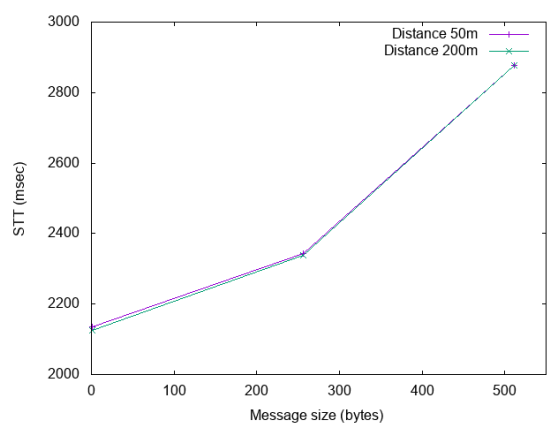

(a)

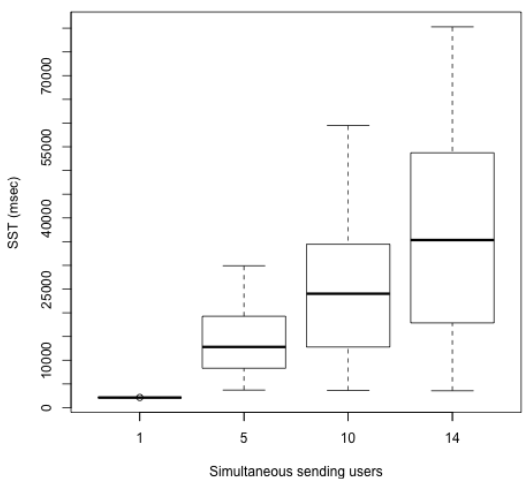

(b)

Fig. 7: Behaviour of the STT when varying the message size (median values) (left). Scalability evaluation of the system by increasing the number of concurrent sending users. (right)

euro. The total cost of the device, including the solar panel, the battery and the enclosure, is about 70 euro.

Finally, regarding regulation, LoRa devices operate in frequencies of $868 \mathrm{MHz}$ and $433 \mathrm{MHz}$ in Europe with $14 \mathrm{dBm}$ maximum output power, and $915 \mathrm{MHz}$ in North and South America, Australia and New Zealand with $20 \mathrm{dBm}$ maximum power; a dozen African countries follow Communications Regulators' Association of Southern Africa (CRASA) Harmonised Frequency Bands For SRD Applications. These frequencies are part of the ISM bands which do not require any license to operate on. To allow for a fair usage of the spectrum, regulators impose a limit on the maximum transmission time allocated to a user in specific frequency. For instance, a 1\% Duty Cycle meaning that the transmitter can only transmit 1\% of the time is in place in Europe, whereas in America the maximum transmission time is $400 \mathrm{~ms}$.

\section{Conclusion}

We have presented a low-cost, low-power consumption solution to provide messaging to isolated communities. We believe that these kind of solutions are necessary since there is still a significant percentage of the world population out of the reach of commercial telecommunication service providers, especially in rural areas of developing countries. Once in place, the LoRa hub can also be used to convey sensors and other kinds of data of interest as well as an early warning system for disasters mitigation.

Our platform integrates a gateway to Telegram, a widely adopted messaging application. This allows extending the reach of the messaging system to the standard Internet. 
We developed a prototype and measured its performance showing its adequacy to the purpose we aimed to. This architecture can be extended and used for other applications like weather forecasting data, health-care information, market prices of crops, disaster prevention and so on.

Improvements of this platform are planned to exploit further possibilities. We are currently working on enabling voice messages to extend the use of the platform to users who are not comfortable with reading and writing. We will also explore the possibility of fitting a bus or other public transportation vehicle that traverses areas not served by the cellular operator with a LoRa hub. This will allow it to gather LoRa text messages that can later be forwarded to the intended destination once the vehicle reaches the coverage of the cellular service. Another important evolution is to provide a mesh structure among hubs in order to extend the reach of the network thus spanning even greater distances. Security and in particular privacy in the communications will also be addressed.

\section{References}

1. A. Pentland R. Fletcher, A.H.: Daknet: rethinking connectivity in developing nations. Computer 37, 78-83 (2004)

2. Ahmad, T., Afutu, A., Adjaho, K., Nyarko, Y., Subramanian, L.: Technical report: Design, implementation and deployment of intermittency-aware cellular edge services for rural areas. arXiv preprint arXiv:1604.05355 (2016)

3. Galati, A., Bourchas, T., Siby, S., Mangold, S.: System architecture for delay tolerant media distribution for rural south africa. In: Proceedings of the 9th ACM International Workshop on Wireless Network Testbeds, Experimental Evaluation and Characterization, WiNTECH '14, pp. 65-72. ACM, New York, NY, USA (2014). DOI 10.1145/2643230.2643239. URL http://doi.acm.org/10.1145/2643230.2643239

4. J. Saldana, A. Arcia-Moret, B. Braem, E. Pietrosemoli, A. Sathiaseelan, and M. Zennaro: Alternative Network Deployments: Taxonomy, Characterization, Technologies, and Architectures. RFC 7962, RFC Editor (2016). URL https://www.rfceditor.org/rfc/pdfrfc/rfc7962.txt.pdf

5. Jovalekic, N., Pietrosemoli, E., Rainone, M., Zennaro, M.: Smart and very distant objects. In: Proceedings of the 3rd Workshop on Experiences with the Design and Implementation of Smart Objects, pp. 29-34. ACM (2017)

6. Martinez, A., Villarroel, V., Seoane, J., del Pozo, F.: Analysis of information and communication needs in rural primary health care in developing countries. IEEE transactions on Information Technology in Biomedicine 9(1), 66-72 (2005)

7. Ntareme, H., Zennaro, M., Pehrson, B.: Delay tolerant network on smartphones: Applications for communication challenged areas. In: Proceedings of the 3rd Extreme Conference on Communication: The Amazon Expedition, p. 14. ACM (2011)

8. Simo-Reigadas, J., Municio, E., Morgado, E., Castro, E.M., Martinez, A.: Sharing lowcost wireless infrastructures with telecommunications operators for backhauling $3 \mathrm{~g}$ services in deprived rural areas. In: 2015 IEEE 16th International Symposium on A World of Wireless, Mobile and Multimedia Networks (WoWMoM), pp. 1-8 (2015). DOI 10.1109/WoWMoM.2015.7158164

9. Vangelista, L., Zanella, A., Zorzi, M.: Long-range iot technologies: The dawn of lora. In: Future Access Enablers of Ubiquitous and Intelligent Infrastructures, pp. 51-58. Springer (2015)

10. Y.-C. Liang A. Hoang, H.H.C.: Cognitive radio on tv bands: a new approach to provide wireless connectivity for rural areas. IEEE Wireless Communications 15(3) (2008) 